\section{Optimizing Infrared Camera Resolution for Small Object Detection using Subpixel Rendering and PIFS in Multiresolution Image Analysis}

\author{
Poerbaningtyas E. ${ }^{1,2 * \odot}$, Dradjat R. S. ${ }^{3}$, Endharti A. T.4, Sakti \\ S. P. ${ }^{5}$, Widjajanto E. ${ }^{6}$, Yueniwati Y. ${ }^{7}$, Purnomo M. H. ${ }^{8}$
}

\begin{abstract}
Background: Breast cancer screening techniques have been developing rapidly in the field of imaging systems. One of these techniques is thermography, which is an alternative modality for mammography to detect breast lesions. Thermography utilization has been progressively developing as various models and methods of object processing improvement. Currently, the Fluke TIS20 infrared camera, with a resolution of $320 \times$ 240 , has not been used to measure precisely small objects such as early breast cancer lesions. Retrieval and processing of single images lead into imprecise object measurements and false positive results.
\end{abstract}

Objective: Problems have been arisen due to the limitations of the camera resolution, object retrieval techniques and suboptimal image processing. The aim of this study was to detect accurately breast cancer lesions in rats, which were induced by carcinogenic compounds.

Material and Methods: In this experimental study, development of models was conducted based on increasing image by optimizing the ability of low-resolution infrared (IR) cameras to identify s mall objects precisely. Image pixel density increased by adjusting the distance of the objects from the camera and multiple images of objects gradually shifting were used to measure object dimensions precisely.

Results: The results showed that cancerous lesions as small as $1.27 \mathrm{~mm}$ could be detected. This method of lesion detection had a sensitivity and specificity of $93 \%$ and $77 \%$ respectively.

Conclusion: Small objects (cancerous lesions) were measured by increasing image resolution through splitting pixels into subpixels and combining several images using Partitioned Iterated Function Systems (PIFS).

Citation: Poerbaningtyas E, Dradjat RS, Endharti AT, Sakti SP, Widjajanto E, Yueniwati Y, Purnomo MH. Optimizing Infrared Camera Resolution for Small Object Detection using Subpixel Rendering and PIFS in Multiresolution Image Analysis. J Biomed Phys Eng. 2020;10(3):261272. doi: $10.31661 /$ jbpe.v0i0.1197.

\section{Keywords}

Measure; Breast Neoplasms; Temperature; Mammography; Screening Cancer; Temperature

\section{Introduction}

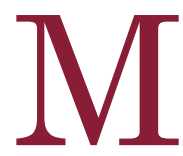
any breast-imaging techniques have been developed as methods, which identify early-stage breast cancer. Imaging technology has been very helpful for doctors in early prevention, diagnosis, and monitoring therapy. One type of medical imaging methods is breast thermography, which uses infrared cameras to detect patterns in breast surface temperature. Higher temperature than the surrounding area is a typical characteristic of a malignant lesion [1]. Ac-
${ }^{1} \mathrm{MT}$, Doctoral Program of Medical Science, Faculty of Medicine, Brawijaya

University, Malang,

Indonesia

${ }^{2} \mathrm{MT}$, Department of In-

formatics, STIKI, Malang,

Indonesia

${ }^{3} \mathrm{PhD}$, Department of

Orthopaedic, Saiful

Anwar Hospital, Faculty

of Medicine, Brawijaya

University, Malang,

Indonesia

${ }^{4} \mathrm{PhD}$, Department of

Parasitology, Faculty

of Medicine, Brawijaya

University, Malang,

Indonesia

${ }^{5} \mathrm{PhD}$, Department of

Physics, Brawijaya Uni-

versity, Malang, Indo-

nesia

${ }^{6} \mathrm{PhD}$, Department of

Clinical Pathology, Fac-

ulty of Medicine, Brawi-

jaya University, Malang,

Indonesia

${ }^{7}$ PhD, Department of

Radiology, Faculty of

Medicine, Brawijaya

University, Malang,

Indonesia

${ }^{8} \mathrm{PhD}$, Department of

Electrical, Faculty of

Electric, ITS, Surabaya,

Indonesia

*Corresponding author:

E. Poerbaningtyas

Doctoral Program of

Medical Science, Faculty

of Medicine, Universi-

ties Brawijaya, Malang,

Indonesia

E-mail: evystudi@gmail.

com

Received: 9 June 2019

Accepted: 12 July 2019 
cording to comparing the effectiveness of the mammography, ultrasonography, and infrared thermography in screening and early diagnosis of breast cancer, there is no difference between the accuracy of mammography and ultrasonography (96.1\% versus $95.8 \%$ ) while the accuracy of infrared thermography is 97.1 . The sensitivity and specificity of infrared thermography is superior to mammography and ultrasonography in lesions with a diameter of less than $2 \mathrm{~cm}$ [2]. Thermographic imaging of the breast combined with mammography and ultrasonography can be used to diagnose lesions as small as $4 \mathrm{~mm}$ [3].

Research in thermographic imaging in breast cancer has been undergoing continuous development through various methods or algorithms. There has been also an ongoing evaluation of the reliability and accuracy of infrared thermography in the assessment of women with breast cancer. Medical imaging techniques have been widely used in the diagnosis and detection of breast cancer. The need to the professional radiologist to establish the diagnosis has been a disadvantage of applying this technique [4]. The application of thermal imaging using an infrared camera can provide maximum information accurately and precisely [5]. Thermography images have been multiresolution images and used "Image of Temperature data" as a basis when processing results produce inconsistent readings [68]. The best input in processing thermography has been provided by the "Raw of Temperature" data obtained from an infrared camera. Another disadvantage of thermography to detect breast cancer lesions is the use of single image. The use of single image data as input will result in less precision in lesion detection so that there will be false positive and negative findings [9-12]. Detection of very small cancerous lesions has been strongly influenced by infrared camera resolution.

Infrared cameras have advantages because of their ability to record objects in image form and allowing all areas of the recorded object to be measured at the same time. On the other hand, due to the limited resolution, the quality of the image has not been sharp frequently. Blurry images can also result from recording a moving/dynamic object producing artefact in the image known as motion artefacts [13]. In infrared thermal cameras, blurry images not only reduce the detail of the object but also correlate with inaccurate temperature measurements.

There are many types and resolutions of infrared cameras, which high-resolution camera is needed to detect small micro-sized objects. Pixel or abbreviation for picture element is the smallest element or frequently called as small colored boxes of a digital image. Resolution is one of the determinants of digital image quality, which is determined by the number and collection of pixels configuring the image. Camera resolution is directly proportional to the image quality [14].

Continually high-quality documentation and reporting will encourage the acceptance of thermography in the clinical setting. The same standard must be applied to routine clinical investigations with thermal imaging. As a noninvasive technique, thermography is easily quantified like infrared imaging [15]. A study conducted in humans has been able to detect as small as $4 \mathrm{~mm}$, but the value of measuring lesion size still has needed to be improved as it has not classified the patient's risk accurately [3]. Typically, while thermography using the Fluke TIS20 infrared camera with 320 $\times 240$ resolution, the results are suboptimal, especially in detecting objects smaller than the pixel box. Camera resolution can be improved by adjusting pixel density, which is dependent on the camera's distance from the recorded object. If an object gets closer to the camera, the pixel density should be the smaller.

This study aims to improve image quality by adjusting the distance between the infrared camera and the malignant breast lesions to increase image resolution and thus improve precision when measuring lesion size. As dis- 
tance adjustment between the camera and the recorded object affects pixel density, a smaller pixel density is obtained when a lesion is closer to the camera. Image resolution is also improved by breaking the pixels into subpixels and performing multi-image analysis. This is combined with partitioned iterated function systems (PIFS), further enhancing the precision of lesion measurement.

The aim of this study is to detect and measure breast cancer lesions of female Wistar rats using the Fluke TIS20 infrared camera. A strength of this study is that the camera used is stable and can be adjusted based on the micrometer, high precision. Several images were combined using PIFS to measure the size of the cancerous lesions.

PIFS is a part of the fractal concepts providing a framework for analyzing natural phenomena in various fields of science such as biology and medicine. Fractal application in analyzing thermographic images can be used to determine breast types based on breast dimensions. The dimensions of normal breasts are smaller than abnormal breasts. PIFS modeling consists of contractual transformation whereas the original image is a fixed point of unification transformation. Each transformation involves a spatial transformation of a square block contract, as well as a linear transformation of the gray level of pixels [16].

Application of PIFS in increasing the contrast of an image is detecting cancer lesions, where PIFS modeling is used to create a low pass version of the original image. Contrastenhanced images are obtained by adding different original images to the low pass version and the original image itself. The low pass version of the original image is obtained by applying repeatedly PIFS to the original image while using lower value for the contrast parameter than specified one [17].

Application of PIFS to improve image contrast and reduce false positive and false negative findings is made. According to the improvements in data collection, increasing image resolution, and PIFS application model in data processing, this study is to provide a new finding to detect cancer lesions in with smallest size. Despite all the limitations of the IR camera, the TiS20 type fluke can increase image resolution, the detection of small objects precisely and reduce the presence of false findings and subjectivity.

\section{Material and Methods}

In this experimental study, due to the Fluke TIS20 infrared camera with 320-by-240 resolution, the results are suboptimal, especially in detecting objects, which are smaller than the pixel box (Figure 1a). Camera resolution can be improved by adjusting pixel density dependent on the camera's distance from the recorded object. If an object gets closer to the camera, the pixel density gets smaller (Figure 1b).

Application of PIFS to increase the contrast of an image causes cancer lesions to be detected as shown in Figure 2. Application of PIFS to improve image contrast and reduce false positive and false negative findings is made (Figure 3). The design of the research device consists of several components that have different roles and functions (Figure 4).

Experimental study to detect small objects was conducted using animal models of female rats. The experiment was carried out with $1.2-1.5$ months rats from Parasitology Laboratory. The rats were induced by DMBA (Dimethylbenz [a] anthracene) carcinogen compounds $(0.4 \mathrm{ml} / \mathrm{gr} / \mathrm{BW}$ with 10 times repetition. After completing carcinogen compound induction, rats were treated for 8 weeks [18]. All experiments were conducted according to guiding principle for scientific purposes and being approved by ethical committee Universities Brawijaya Malang Indonesia (884-KEP-UB).

IR camera was used as a temperature sensor to record temperature of animal model body. The IR camera used was Fluke TiS20-9Hz type. This camera has an FPA (Focus Plane 
(a)

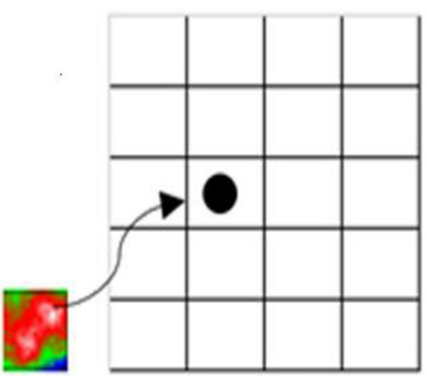

(b)

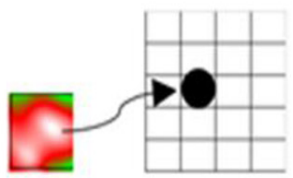

Figure 1: a) The size of an object is smaller than a pixel box so that it cannot measure the size of an object precisely. b) The effect of setting the retrieval distance of an object will reduce pixel density so that the size of the object can be measured at least the size of a pixel box.

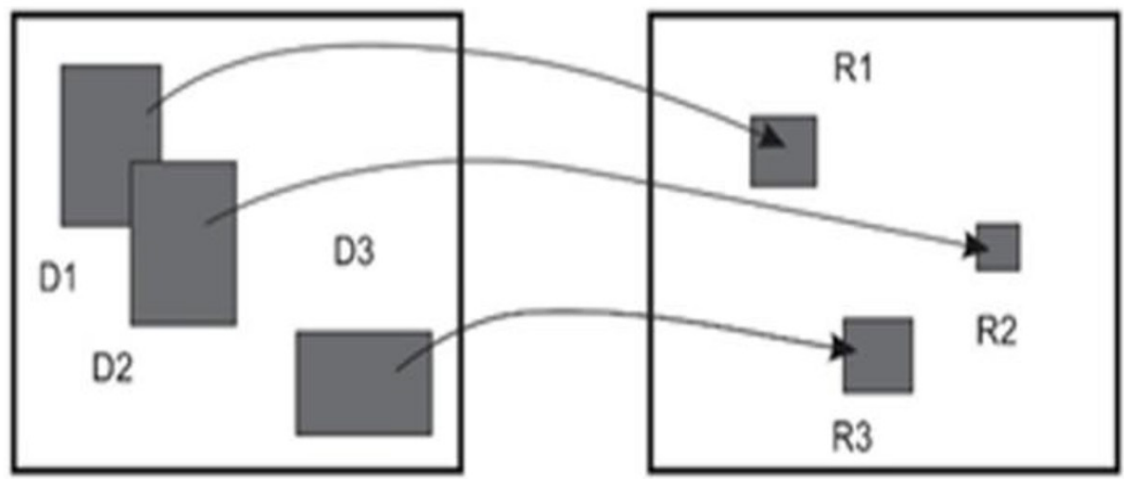

Figure 2: Mapping of large and small squares in PIFS [15]. The definition of PIFS is in the form of an image, where large squares are a combination of small squares overlapping with each other.

(a)

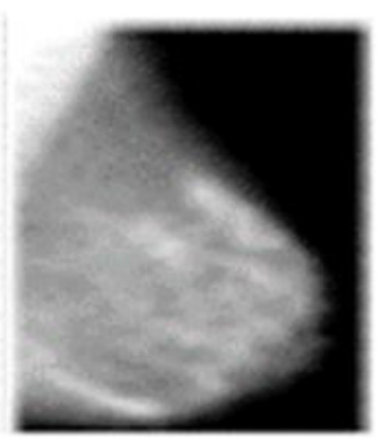

(b)

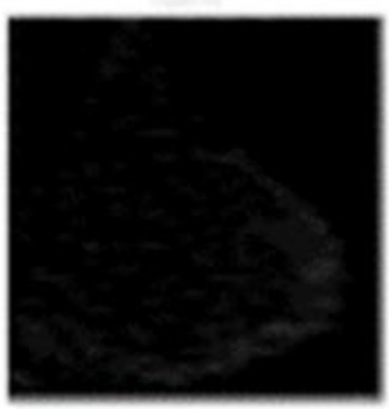

Figure 3: a) Thermography results b) contrast enhancement of imaging using PIFS [16]. Thermographic output in the form of temperature image and lesion reading were performed by increasing image contrast using PIFS. 
(a)

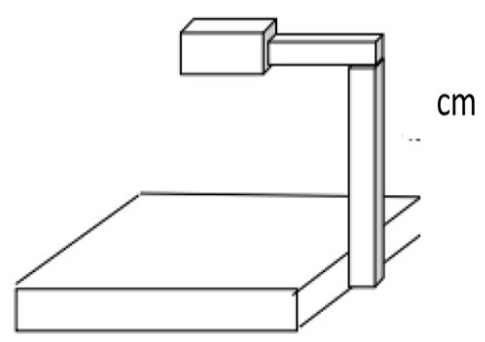

(b)

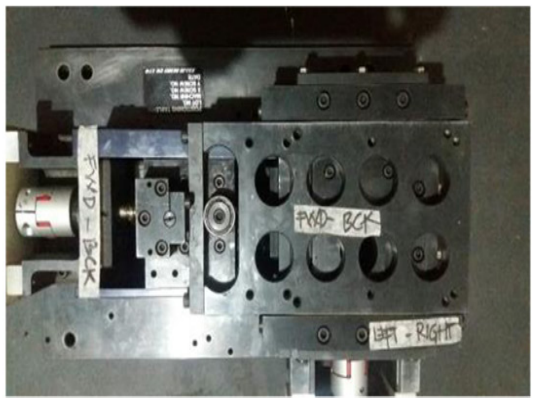

Figure 4: a) Camera supporting to adjust the distance between the camera and the object causes the optimal pixel resolution of the camera resolution to be better.

b) "XY linear "table is to shift object right, left, back and forth, and get many images.

Array) type of uncooled microbolometer vanadium oxide (VOX) material, with a $7.5 \mathrm{~Hz}$ refresh rate and works on the spectral band 8-14 $\mu \mathrm{m}$. In addition, this camera has digital display resolution of $320 \times 240$ pixels and measurement range of $-20^{\circ} \mathrm{C}$ to $600^{\circ} \mathrm{C}$ with + $2{ }^{\circ} \mathrm{C} /+2$ percent accuracy.

Stick was used as a camera support to stabilize camera. This stick was a variable that affects pixel density and has a function to adjust the distance between animal model and camera (Figure 4a). Wax platform was used to provide cold temperatures so that the heat of objects can be measured perfectly.

"XY Linear" table (Figure 4b) was used to move immobile rats and slide 5 steps of left and right and also back and forth, with a very small shift with high resistance. The specifications of the "XY Linear" table are equipped with a stepper motor with 2000 step per pulse resolution; repeatability reaches $0.02,1 \%$ of stepper motor error, which are not accumulative in 1 conversion cycle with a $5 \mathrm{~mm}$ shift to maintain the movement consistency of the "XY Linear" shift table and a thelso gradual movement is controlled by a microcontroller. A microcontroller to maintain the consistency of each step motor movement controls the linear table. The movement model is to have 25 of different positions, with $0.25 \mathrm{~mm}$ shift per step. Shift parameters are set with a maximum reference of $50 \%$ of pixel density and the width of the pixel box in the picture $0.475 \mathrm{~mm}$.

The heat body temperature of each rats was measured every week using an IR camera. This study is classified into 2 procedures: the first step to retrieve temperature of lesion and the second one to measure the size of lesion for processing image.

The first procedure consists of some items, as follows: 1) adjusting distance stick between animal model and camera to have optimal resolution. 2) Anesthetizing rats with ketamine intraperitoneal. 3) Immobilizing animal model on the table "XY Linear". 4) Focusing on adjustment of central position of animal model body. 5) Collecting data by capturing repeatedly as many as $25 \mathrm{x}$ with different positions, by shifting the "XY Linear" table right / left and forward / backward. The second procedure follows 5 steps:

1) Data from an IR camera (dot ISV) changed to "Raw of Temperature (dot txt)".

2) The size of lesion was measured using single image then followed using multi-image.

3) Image resolution changed using subpixel rendering (340-by-240 to 640-by-480 matrix).

4) 25 images were merged into 1 image with PIFS.

5) The lesion area was cropped (16-by-16 
matrix) and followed by measuring the length and width of lesion.

\section{Results}

Thermography is a non-invasive test mapping the thermal patterns on the surface of objects. It has the benefit of evaluating an object based on a distance and preserving the object's structural integrity [19].

Initially, in a single image analysis, the variation of distance between the camera and the object is tested. The results were obtained at a distance of $10 \mathrm{~cm}$ between the camera and the object, with a cross-sectional and pixel density

(a)

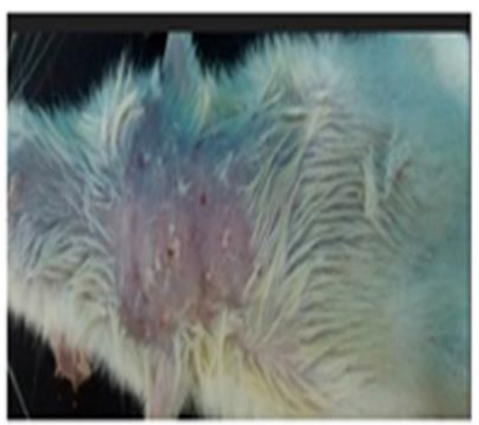

width of $15.21 \mathrm{~cm}$ and $0.475 \mathrm{~mm}$, respectively. The shorter capturing distance between the camera and the object reduced the pixel density and allowed small objects to be detected and measured (Figure 5 and Table 1).

Based on analyzing single thermal image with a resolution of $340 \times 240$, the smallest object size (malignant breast lesion) was detected at $5.4 \times 6.9 \mathrm{~mm}$ in rat 5 . Average of detection is at week 4 in rat-3, rat-5 and rat-7. In rat-9, a $7.5 \times 6.3 \mathrm{~mm}$ lesion was detected, but this was only identified 8 weeks after carcinogen administration. Using thermography as a screening tool is safer and more effective in

(b)

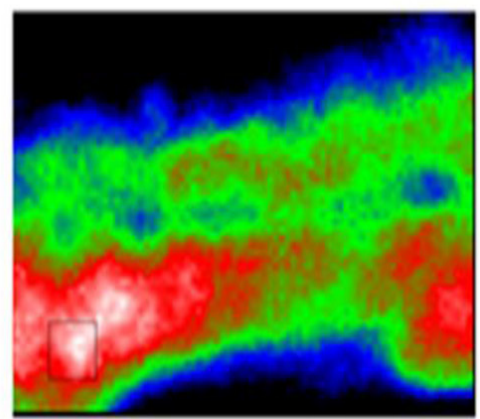

Figure 5: a) Visual Rat: location of breast cancer in research objects.

b) Thermal imaging of rat heat information on an object in the form of images, where white shows the highest temperature.

Table 1: Result of cancer lesions measurement with 1 image ( $1^{\text {nd }}$ model).

\begin{tabular}{ccc} 
Rat Code & Lesion Size Detected $(\mathbf{m m})$ & Detected Rata Was Found in Lesions \\
\hline Rat -3 & $7.5 \times 7.5$ & Week - 4 \\
\hline Rat -5 & $5.4 \times 6.9$ & Week - 4 \\
\hline Rat -7 & $7.5 \times 7.5$ & Week - 4 \\
\hline Rat -8 & $6.6 \times 5.5$ & Week - 7 \\
\hline Rat -9 & $7.5 \times 6.3$ & Until the $8^{\text {th }}$ week there is no cancer lesions \\
\hline Rat -11 & $9.6 \times 9.6$ & Week - 8 \\
\hline Rat -12 & $6.45 \times 7.5$ & Week - 5 \\
\hline Rat -16 & $7.5 \times 4.5$ & Week - 6
\end{tabular}


the setting of breast cancer. The resolution of infrared cameras is much lower than optical cameras so that most infrared cameras only have a resolution of $160 \times 120$ or $320 \times 240$ pixels. The reading resistance is low and needs improvement. In this study, a method was introduced to increase image resolution of an infrared camera. Measuring small objects in multiresolution images was conducted by not only increasing the image resolution through increasing pixel density but also changing the image dimensions, further improving image resolution.

In the multi-image analysis process, the standard resolution of 320-by-240 matrix of the Fluke TIS20 camera also increased by break- ing pixels into subpixels causing the dimensions of the image to change into 640-by-480 matrix. Subpixel modeling aimed to increase the IR camera resolution from 340-by-240 to 640-by-480 matrix, and Figure 6 displays the subpixel. However, the value of heat intensity in each pixel does not change using the subpixel technique; the level of image resolution can increase up to 2 folds, from $320 \times 240$ to $640 \times 480$. The pixel value of a subpixel fraction is the same as the individual subpixel, and Figure 7 displays the pixel.

When the position of an object is in a pixel intersection (Figure 8), it is difficult to identify the size of the object precisely due to the frequent reading done in a single image and

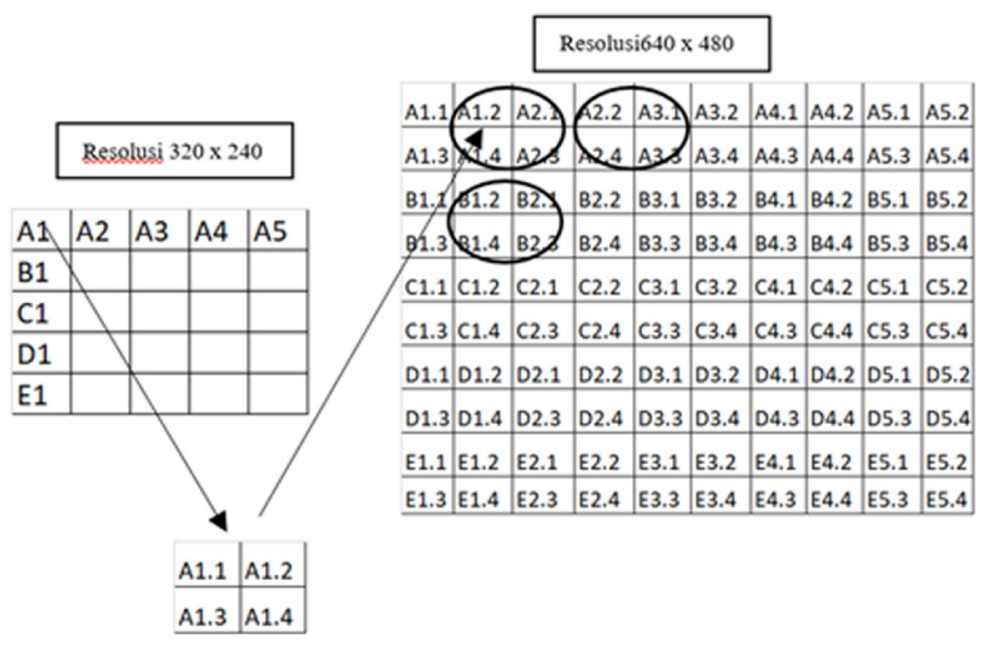

Figure 6: Subpixel creation: the image describes the creation of a subpixel, which breaks 1 pixel into 4 pixels. The hot temperature of the pixel will be copied to 4 pixel fractions, so that the original image resolution changes from $320 \times 240$ to $640 \times 480$.

\begin{tabular}{|r|r|r|r|}
30.9 & 30.98 & 31.05 & 31.1 \\
\hline 31 & 31.01 & 31.06 & 31.1 \\
\hline 31.1 & 31.06 & 31.09 & 31.1 \\
\hline 31.1 & 31.11 & 31.12 & 31.1 \\
\hline
\end{tabular}

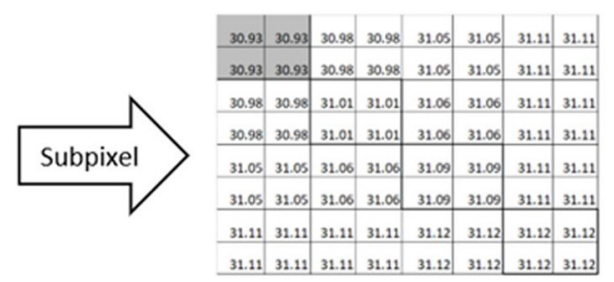

Figure 7: Subpixel creation. The image describes the value of each pixel in subpixel with the same value. 
generating a wide reading area of the object. Improvements with PIFS using several images (multi images) produce readings with good precision. The use of the "XY Linear" shift table in object collection is helpful in producing many images with very small differences
(Figure 9a). PIFS is a technique combining multiple overlapping images to obtain 1 single image, as portrayed in Figures $9 b$ and 10.

After merging these 25 images into 1 image, the size of the object was measured by cropping a $16 \times 16$ pixel block from the high-

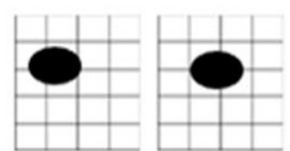

(1st) $(2 n d)$

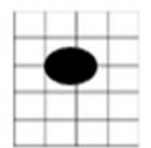

(3th)

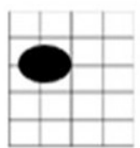

(4th)

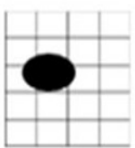

(5th)

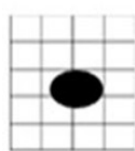

(6th)

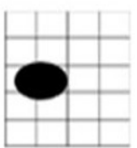

(25th)

Figure 8: The position of an object at a pixel intersection and data collection is repeated up to 25 times where the shift per image is $0.23 \mathrm{~mm}$ using the "XY Linear" table.

a)

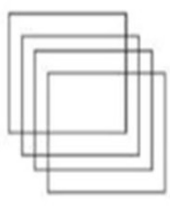

b)
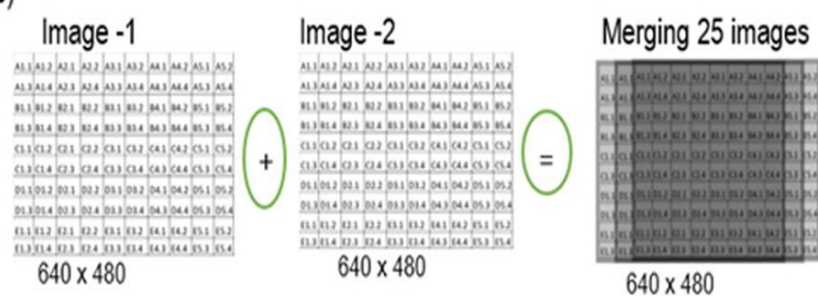

$640 \times 480$

Figure 9: Combining 25 images into 1 image shows the determination of precision by applying the PIFS method merging 25 images (multi images) into 1 image, with an average value of hot temperature degrees in each pixel per image.

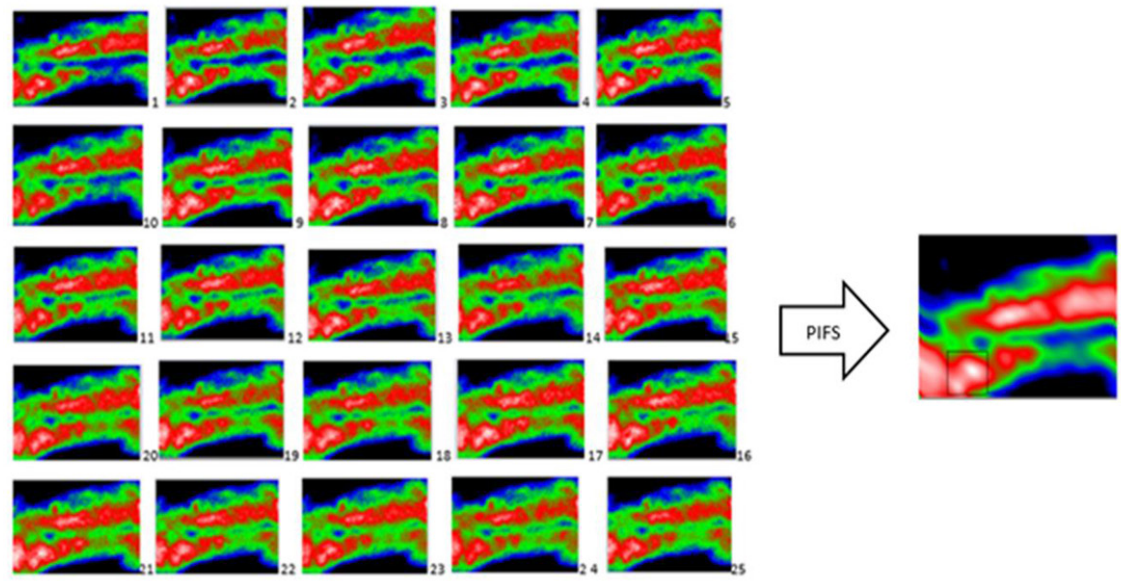

Figure 10: Combining 25 images into 1 image using PIFS modeling. 
est temperature area. Heat pattern segmentation was applied on the cropped segment to determine the size of the object in 2 dimensions (length and width). Pixel density, as determined by the $10 \mathrm{~cm}$ distance between the camera and the object, was $0.475 \mathrm{~mm}$ per pixel. Once subpixel rendering and PIFS was applied to merge image, the coupling density became $0.235 \mathrm{~mm}$ enabling the measurement of very small objects precisely. In this study, a measurement of cancerous lesions was conducted using subpixel rendering and PIFS to increase image resolution and allow small objects to measure precisely (Table 2).

Owing to merging 25 images and applying subpixel rendering and PIFS, clear visualization of the breast cancer lesions is caused, which leads into precise measurements. Taking numerous images of the object will significantly increase accuracy when measuring the size of the cancerous lesion; thus, it leads into early detection of malignancy.

\section{Discussion}

Based on the results of this study, when applying standard capturing and processing techniques and using only 1 image, the smallest lesion that can be detected is $5.4 \mathrm{~mm}$. However, using multiple images, adjusting the distance between the object and the camera and increasing the image resolution with subpixel rendering and PIFS, cancerous lesions as small as $1.27 \mathrm{~mm}$ can be detected. This translates into an improvement in lesion detection of $56.77 \%$.

Treatment of rats for 8 weeks will affect the increase in temperature on the breast area and the development of lesion. Long-term incubation of carcinogens will affect higher temperature in the breast area and result in the development of larger cancer lesions. There exists a linear correlation between the incubation period of the carcinogens and the temperature and size of the malignant breast lesions. The rise in temperate and rate of lesion development varied from one rat to another one, but the study clearly demonstrated a strong correlation with a constant value of 0.1776 , which Figure 11 presents the rise.

In addition to measuring the cancerous lesions, this processing technique was also able to establish a system for early detection. The early detection rate was obtained using the temperature and comparing the highest temperature with the average temperature in the breast area. The difference between the highest and average temperature indicated the potential for the emergence of a cancerous lesion. Analyzing the growth rate of cancerous lesions in one image compared to multiple im-

Table 2: Result of cancer lesions measurement with many images and the application of subpixel and PIFS modeling ( $2^{\text {nd }}$ model).

Rat Code Lesion Size Detected $(\mathrm{mm}) \quad$ Detected Rata Was Found in Lesions

\begin{tabular}{|c|c|c|}
\hline Rat -3 & $1.27 \times 1.72$ & Week - 4 \\
\hline Rat -5 & $2.85 \times 3.5$ & Week - 3 \\
\hline Rat -7 & $2.02 \times 3.7$ & Week - 3 \\
\hline Rat - 8 & $3.7 \times 3.7$ & Week - 3 \\
\hline Rat -9 & $4.5 \times 4.5$ & Week - 1 \\
\hline Rat - 11 & $3.28 \times 3.75$ & Week - 2 \\
\hline Rat -12 & $2.32 \times 3.75$ & Week - 2 \\
\hline Rat -16 & $3.42 \times 3.75$ & Week - 4 \\
\hline
\end{tabular}




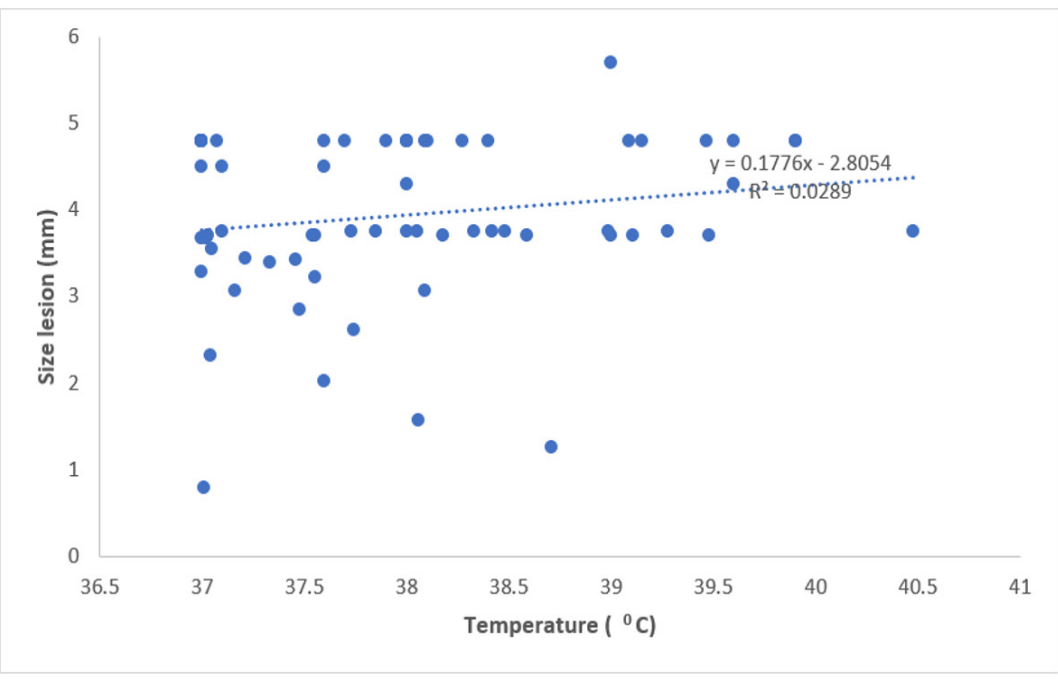

Figure 11: Graph of temperature relations with the size of cancer lesions. The figure shows a strong correlation between high temperatures proportional to the size of cancer lesions.

ages produces different growth rates. Analyzing lesion growth rate using many images can provide information that is more accurate and faster.

In rat-5 and rat-7, when processing only 1 thermal image, a malignant lesion was only detected in the $4^{\text {th }}$ week. However, when multiple images were processed from the same rats, malignant lesions were already detected in the $3^{\text {rd }}$ week. In the $9^{\text {th }}$ rat there were no cancer lesions, but in multiple image with the appli- cation of subpixel modeling and PIFS, cancer lesions were found in the first week. Overall, there was an $80 \%$ increase in the detection of cancerous lesions. Overall, there was an increase of $80 \%$ in the detection of cancerous lesions (Table 3).

In Table 3 of the second model, there is an improvement in the size of the smallest lesions detected, number of cancerous lesions identified, sensitivity and specificity. This is demonstrated by the increase of $58 \%$ to $93 \%$

Table 3: Results of processing research objects between first model and second models.

Testing

\begin{tabular}{ccc}
\hline $\begin{array}{c}\text { The smallest object size } \\
\text { detected }\end{array}$ & $5.4 \times 6.9 \mathrm{~mm}$ & $1.27 \times 1.72 \mathrm{~mm}$ \\
\hline $\begin{array}{c}\text { Speed of early detection } \\
\text { of cancer lesions }\end{array}$ & Week-4 & Week-1 \\
\hline Sensitivity & $58 \%$ & $93 \%$ \\
\hline Specification & $33 \%$ & $77 \%$ \\
\hline Accuracy & $43 \%$ & $80 \%$
\end{tabular}


in sensitivity and $33 \%$ to $77 \%$ in specificity from the first model to the second one. This review provides the basic and rational principles for ranking the analytical methods and results of continuous (ROC) diagnostic tests as the gold standard [20]. In the graph showing the distribution of pixel heat intensity, the second model is represented by a periodic graph with continuous repetition, while in the first model this is absent (Figure 12). a)

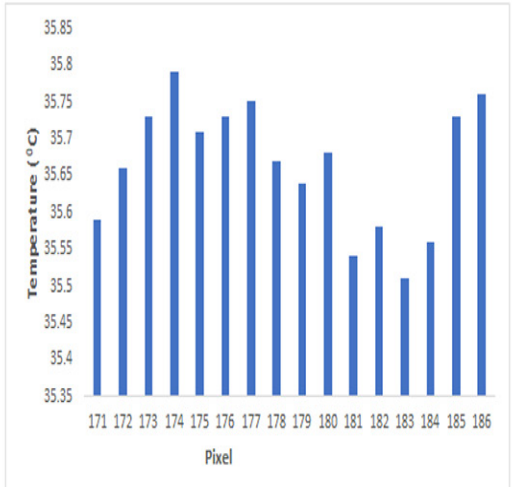

b)

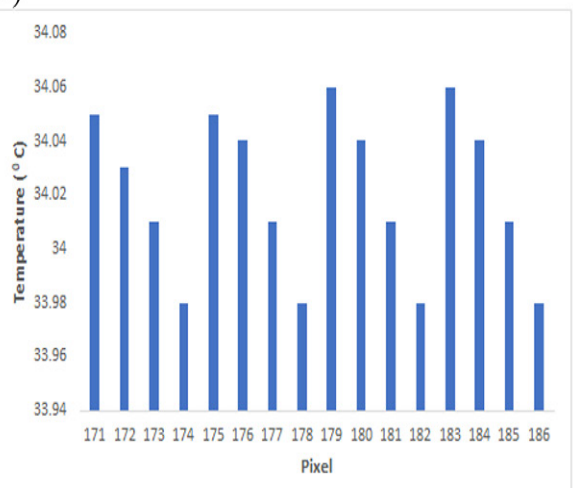

Figure 12: a) Temperature distribution in first model. b) Temperature distribution in the second model.

\section{Conclusion}

In this study, the limitations of resolution, the Fluke TiS20 $340 \times 240$ camera in small object detection, can be solved using subpixel rendering and PIFS method. The results reveal that the smallest cancer lesions of $1.27 \mathrm{~mm}$ in size can be detected in the first week with $93 \%$ and $77 \%$ of sensitivity and specificity, respectively.

\section{Acknowledgment}

The author would like to thank Universitas Brawijaya laboratory staff on behalf of $\mathrm{Mr}$. Ridha who helped complete this research.

\section{Conflict of Interest}

None

\section{References}

1. Han F, Liang CW, Shi GL, Wang L, Li KY. Clinical applications of internal heat source analysis for breast cancer identification. Genet Mol Res. 2015;14:1450-60. doi: 10.4238/2015.Febru- ary.13.24. PubMed PMID: 25730084.

2. Yao X, Wei W, Li J, Wang L, Xu Z, Wan Y, et al. A comparison of mammography, ultrasonography, and far-infrared thermography with pathological results in screening and early diagnosis of breast cancer. Asian Biomedicine. 2014;8:11-9. doi: 10.5372/1905-7415.0801.257.

3. Arena F, Barone C, DiCicco T, editors. Use of digital infrared imaging in enhanced breast cancer detection and monitoring of the clinical response to treatment. Proceedings of the 25th Annual International Conference of the IEEE Engineering in Medicine and Biology Society (IEEE Cat. No.03CH37439); Cancun, Mexico: IEEE; 2003. doi: 10.1109/IEMBS.2003.1279447.

4. Mehdy MM, Ng PY, Shair EF, Saleh NIM, Gomes C. Artificial Neural Networks in Image Processing for Early Detection of Breast Cancer. Comput Math Methods Med. 2017;2017:2610628. doi: 10.1155/2017/2610628. PubMed PMID: 28473865. PubMed PMCID: PMCPMC5394406.

5. Kennedy DA, Lee T, Seely D. A comparative review of thermography as a breast cancer screening technique. Integr Cancer Ther. 2009;8:9-16. doi: 10.1177/1534735408326171. PubMed PMID: 19223370. 
6. Whiteford JK, Whiteford JR. Screening for breast cancer. Ann Intern Med. 2003;138:768. doi: 10.7326/0003-4819-138-9-200305060-00020.

7. Ghayoumi Zadeh $\mathrm{H}$, Haddadnia J, Montazeri A. A Model for Diagnosing Breast Cancerous Tissue from Thermal Images Using Active Contour and Lyapunov Exponent. Iran J Public Health. 2016;45:657-69. PubMed PMID: 27398339. PubMed PMCID: PMCPMC4935710.

8. Zadeh HG, Haddadnia J, Zadeh FJS, Zadeh ZE, Kianersi S, Masoumzadeh S, et al. A review of the dedicated studies to breast cancer diagnosis by thermal imaging in the fields of medical and artificial intelligence sciences. Biomedical Research. 2016;27:543-52.

9. Serrano RC, Motta L, Batista M, Conci A, editors. Using a new method in thermal images to diagnose early breast diseases. XXIInd Brazilian Symposium on Computer Graphics and Image Processing - SIBGRAPI; Rio De Janeiro, Brazil; 2009.

10. Prabha S, Suganthi SS, Sujatha CM. An approach to analyze the breast tissues in infrared images using nonlinear adaptive level sets and Riesz transform features. Technol Health Care. 2015;23:42942. doi: 10.3233/THC-150915. PubMed PMID: 26409908.

11. Francis SV, Sasikala M, Saranya S. Detection of breast abnormality from thermograms using curvelet transform based feature extraction. J Med Syst. 2014;38:23. doi: 10.1007/s10916-0140023-3. PubMed PMID: 24659445.

12. Kermani S, Samadzadehaghdam N, EtehadTavakol M. Automatic color segmentation of breast infrared images using a Gaussian mixture mod- el. Optik. 2015;126:3288-94. doi: 10.1016/j. ijleo.2015.08.007.

13. Malczewski K, Stasinski R. Super-Resolution Thermal Image Reconstruction. 17th International Conference on Systems, Signals and Image Processing; Rio De Janeiro, IWSSIP; 2010.

14. S. Committee. CIPA DCG-001-Translation-2005. Guidel Camera Imaging Prod Assoc; 2005. p. $1-21$.

15. Ring E, Ammer K. The technique of infrared imaging in medicine. Chapter 1. Bristol: IOPScience; 2015. P. 1-10.

16. Mitra S, Murthy C, Kundu M. Partitioned iterative function system: A new tool for digital imaging. IETE Journal of Research. 2000;46:279-98. doi: 10.1080/03772063.2000.11416168.

17. Economopoulos TL, Asvestas PA, Matsopoulos GK. Contrast enhancement of images using partitioned iterated function systems. Image and vision computing. 2010;28:45-54.

18. Kubatka P, Ahlersova E, Ahlers I, Bojkova B, Kalicka $\mathrm{K}$, Adamekova $\mathrm{E}$, et al. Variability of mammary carcinogenesis induction in female SpragueDawley and Wistar: Han rats: the effect of season and age. Physiol Res. 2002;51:633-40. PubMed PMID: 12511189.

19. Thajeel HA. Numerical modeling of infrared thermography techniques via ANSYS. Missouri S\&T; 2013.

20. Hajian-Tilaki K. Receiver Operating Characteristic (ROC) Curve Analysis for Medical Diagnostic Test Evaluation. Caspian J Intern Med. 2013;4:627-35. PubMed PMID: 24009950. PubMed PMCID: PMCPMC3755824. 